\title{
Microstructural response of a near-critical colloid-polymer mixture to shear flow
}

\author{
M. P. Lettinga, H. Wang,* and J. K. G. Dhont \\ IFF, Institut Weiche Materie, Forschungszentrum Jülich, D-52425 Jülich, Germany \\ (Received 27 July 2004; published 13 December 2004)
}

\begin{abstract}
Long-ranged microstructural order of a near-critical colloid-polymer mixture under stationary and oscillatory shear flow is studied by means of time-resolved small-angle light scattering. The distance from the critical point, the shear rate, and the frequency of oscillation are systematically varied. Unexpected shear-induced distortions of critical microstructural order is observed in directions perpendicular to the flow direction, more so on closer approach of the gas-liquid critical point. The measured distortion of long-ranged order can be quantitatively understood on the basis of an approximate solution of the Smoluchowski equation, provided that a nonanalytical distortion of short-ranged microstructural order is assumed. These short-ranged microstructural changes, induced by the flow, account for the observed distortion perpendicular to the flow direction, and are responsible for the shear-induced shift of the location of the gas-liquid critical point. The importance of short-ranged correlations renders the behavior of critical systems under shear flow nonuniversal. The origin of the nonanalytic dependence of short-ranged distortions on the shear rate remains unclear. Including these short-ranged microstructural distortions, we find a good agreement between theory and experiment for both stationary and oscillatory shear flow.
\end{abstract}

DOI: 10.1103/PhysRevE.70.061405

PACS number(s): 82.70.Dd, 05.70.Jk, 05.70.Ln, 83.60.Df

\section{INTRODUCTION}

The effect of external fields, shear flow in particular, on the phase behavior of colloidal systems has been of increasing interest during recent years (see citations [1-3] for reviews). Of special interest are colloidal systems since these are experimentally more easily accessible as compared to molecular systems because of the much larger time and length scales involved. Moreover, the intercolloidal particle potential can be varied from steeply attractive to long-ranged repulsive, leading to very different types of phase transitions and nonequilibrium states like gels and aggregates. Several experimental techniques have been developed to study the flow behavior of both the macroscopic and microscopic structure in situ. Aggregation of attractive spheres was studied by confocal microscopy [4], two-dimensional video microscopy [5], and light scattering [6]. Crystallization of charge or sterically stabilized spheres was studied by means of rheology combined with small-angle neutron scattering [7] or small-angle X-ray scattering [8], respectively.

Where the effect of shear flow on gelation, aggregation, crystallization, and also spinodal decomposition [9-16] has been studied extensively, relatively little is known about the effect of shear flow on systems close to the gas-liquid critical point, just before spinodal decomposition sets in. On approach of the gas-liquid critical point, interactions become very long ranged. In addition, the gradient diffusion coefficient tends to zero so that the dynamics of the system severely slows down, often referred to as critical slowing down. Due to the long-ranged spatial correlations and their very slow dynamics, the microstructural order near the gasliquid critical point is very sensitive to shear flow [1,17-19].

\footnotetext{
*Present address: Department of Physics, North Dakota State University, Fargo, North Dakota, USA.
}

Nonlinear response to either stationary or oscillatory flow is therefore expected to occur at very low shear rates. The outof-phase response of microstructural order to oscillatory flow, responsible for the viscoelastic behavior of near-critical suspensions, occurs at relatively low frequencies.

The effect of shear on near-critical systems has been studied for binary fluids [20,21], polymer blends [22-24], and polymer [25-27] and micellar solutions [28]. In the present paper we investigate, both theoretically and experimentally, the response of a near-critical colloid-polymer mixture to stationary and oscillatory shear flow. Thus we pursue the line set by earlier scattering experiments on critical dispersions of attractive colloids ${ }^{29-33}$ under shear. Having established the link between the experimentally determined and theoretically calculated microstructure, one can continue to predict rheological behavior [18], since it has been shown that shearinduced distortions of the critical structure factor of such dispersions are the origin of near-critical viscoelastic behavior [30].

The effect of shear flow on macroscopic properties is often described on the basis of thermodynamics [14-16,34]. However, in principle no Hamiltonian or free energy can be defined for a sheared system, which has been nicely demonstrated by simulations on nucleation of crystals under shear [35]. The response of colloidal dispersions to shear flow must therefore be studied on the basis of kinetic equations, e.g., the Smoluchowski equation, which is an equation of motion for the probability density function of the position coordinates of the colloidal particles. In addition, for nearcritical systems, nonlinear response should be described by theory, since the long-ranged structures and slow dynamics limits linear response to only very small shear rates. There are a few theoretical approaches for microstructural order under flow for simple fluids [36-40]. Here we shall consider a theory that applies to near-critical dispersions of colloidal spheres, that has been developed by one of the authors (J.K.G.D.) $[32,41]$. In this theory, it is assumed that the 
shear-induced distortions of short-ranged microstructural order can be neglected. That is, it is assumed that the paircorrelation function for small separations between colloidal particles (at most equal to the range of the bare pairinteraction potential) is not affected by shear flow for the small shear rates that are needed to perturb critical longranged structure. As will turn out in the present study, however, this neglect is not justified, and data can be interpreted on the basis of this theory only if it is extended to include such short-ranged distortions. It has been shown before that these short-ranged distortions are responsible for the shearinduced shift of the gas-liquid critical point [42]. Hence, very close to the critical point, a small shift is already sufficient to substantially affect long-ranged order. What is needed in this extension to explain the experimental data is a leading order isotropic distortion that is proportional to the absolute value of the shear rate. If one assumes that the pair-correlation function for small separations between colloidal particles is an analytic function of the shear rate, for symmetry reasons, the leading order isotropic correction should vary like the shear rate squared. The nonanalytic behavior that is found in the present study is therefore unexpected and remains unexplained. A direct verification of the nonanalytic shear-rate dependence of the location of the critical point has not yet been undertaken.

Here we perform in situ time-resolved small-angle light scattering measurements in the velocity-vorticity plane on near-critical colloid-polymer mixtures, systematically varying the shear rate under stationary flow and the frequency and strain amplitude under oscillatory flow. The system that is used consists of colloidal hard spheres, where a depletion attraction is induced by adding polymer to the solvent $[43,44]$. At sufficiently high concentrations of polymer, a gas-liquid phase separation occurs. The distance to the critical point, as measured by the correlation length of the unsheared, quiescent system, can easily be tuned and the critical structure factor is accessible with small-angle light scattering $[30,33]$.

We shall first discuss in Sec. II the theoretical prediction for the shear-rate and frequency dependence of the OrnsteinZernike structure factor, with the neglect of shear-induced short-ranged distortions. It is then shown why the shear-rate dependence of the pair-correlation function for short distances becomes important on approach to the critical point. The theory is extended in Sec. III to include these shortranged distortions. Experimental data are then presented in Sec. IV, which are compared to both the older version of the theory and the extended theory. The paper concludes with a summary and conclusions.

\section{THEORETICAL PREDICTION WITHOUT DISTORTIONS OF SHORT-RANGED CORRELATIONS}

In this section, an existing theory $[32,41]$ on the shearinduced distortion of the Ornstein-Zernike structure factor will be briefly reviewed. One of the assumptions involved in deriving an explicit expression for the structure factor is incorrect on close approach to the critical point, and will be reconsidered in the subsequent section.
The flow that will be considered here is a laminar flow with spatially uniform shear rate $\dot{\gamma}$. The flow velocity $\mathbf{u}$ is chosen as

$$
\mathbf{u}=\dot{\gamma} \hat{\boldsymbol{\Gamma}} \cdot \mathbf{r},
$$

where the normalized velocity gradient tensor $\hat{\boldsymbol{\Gamma}}$ is equal to

$$
\hat{\boldsymbol{\Gamma}}=\left(\begin{array}{lll}
0 & 1 & 0 \\
0 & 0 & 0 \\
0 & 0 & 0
\end{array}\right) \text {. }
$$

This is a flow in the $x$ direction, with its gradient in the $y$ direction, while the $z$ direction is the vorticity direction. In case of stationary shear flow, the shear rate $\dot{\gamma}$ is a constant, while for oscillatory shear flow,

$$
\dot{\gamma}=\dot{\gamma}_{0} \cos \{\omega t\},
$$

where $\omega$ is the frequency of oscillation.

The starting point for the derivation of an equation of motion for the pair-correlation function is the Smoluchowski equation. This is the equation of motion for the probability density function $P \equiv P\left(\mathbf{r}_{1}, \ldots, \mathbf{r}_{N}, t\right)$ of the position coordinates $\mathbf{r}_{j}, j=1, \ldots, N$, of the $N$ spherical macromolecules in the system under consideration. With the neglect of hydrodynamic interactions, the Smoluchowski equation reads [45-47]

$$
\frac{\partial P}{\partial t}=D_{0} \sum_{j=1}^{N} \boldsymbol{\nabla}_{j} \cdot\left[\boldsymbol{\nabla}_{j} P+\beta P \boldsymbol{\nabla}_{j} \Phi\right]-\dot{\gamma} \sum_{j=1}^{N} \boldsymbol{\nabla}_{j} \cdot\left[P \hat{\boldsymbol{\Gamma}} \cdot \mathbf{r}_{j}\right] .
$$

Here, $D_{0}$ is the Einstein diffusion coefficient for a single, noninteracting macromolecule, $\boldsymbol{\nabla}_{j}$ is the gradient with respect to $\mathbf{r}_{j}, \beta=1 / k_{B} T$ ( $k_{B}$ is the Boltzmann constant and $T$ is the temperature), and $\Phi$ is the total potential energy of the assembly of $N$ macromolecules (averaged over the phase space coordinates of the solvent molecules). An equation of motion for the pair-correlation function $g$ can be found by integration, since for homogeneous systems with number density $\rho$, by definition,

$$
\begin{aligned}
P_{2}\left(\mathbf{r}_{1}, \mathbf{r}_{2}, t\right) & =\frac{\rho^{2}}{N^{2}} g\left(\mathbf{r}_{1}-\mathbf{r}_{2}, t\right) \\
& =\int d \mathbf{r}_{3} \cdots \int d \mathbf{r}_{N} P\left(\mathbf{r}_{1}, \mathbf{r}_{2}, \mathbf{r}_{3}, \ldots, \mathbf{r}_{N}, t\right),
\end{aligned}
$$

where $P_{2}$ is the two-particle probability density function. Assuming a pairwise additive interaction potential, that is,

$$
\Phi=\sum_{i>j}^{N} V\left(\left|\mathbf{r}_{i}-\mathbf{r}_{j}\right|\right),
$$

where $V$ is the bare pair-interaction potential, integration of the Smoluchowski equation (4) leads to (with $\mathbf{r}=\mathbf{r}_{1}-\mathbf{r}_{2}$ the distance between two particles and $\boldsymbol{\nabla}$ the gradient operator with respect to $\mathbf{r}$ ) 


$$
\begin{aligned}
\frac{\partial g(\mathbf{r}, t)}{\partial t}= & 2 D_{0} \boldsymbol{\nabla} \cdot\left[\boldsymbol{\nabla} g(\mathbf{r}, t)+\beta g(\mathbf{r}, t)\left\{\boldsymbol{\nabla} V(r)-\mathbf{F}^{i n d}(\mathbf{r}, t)\right\}\right] \\
& -\dot{\gamma} \boldsymbol{\nabla}[g(\mathbf{r}, t) \hat{\boldsymbol{\Gamma}} \cdot \mathbf{r}] .
\end{aligned}
$$

Here, the force between two particles that is mediated via other particles, the "indirect force," is given by (with $\nabla^{\prime}$ the gradient operator with respect to $\mathbf{r}^{\prime}=\mathbf{r}_{1}-\mathbf{r}_{3}$ ),

$$
\mathbf{F}^{\text {ind }}(\mathbf{r}, t)=-\rho \int d \mathbf{r}^{\prime}\left[\boldsymbol{\nabla}^{\prime} V\left(\mathbf{r}^{\prime}\right)\right] \frac{g_{3}\left(\mathbf{r}, \mathbf{r}^{\prime}, t\right)}{g(\mathbf{r}, t)},
$$

where $g_{3}$ is the triplet correlation function, which is defined as, similarly to $g$ in Eq. (5),

$$
\frac{\rho^{3}}{N^{3}} g_{3}\left(\mathbf{r}_{1}, \mathbf{r}_{2}, \mathbf{r}_{3}, t\right) \equiv \int d \mathbf{r}_{4} \cdots \int d \mathbf{r}_{N} P\left(\mathbf{r}_{1}, \mathbf{r}_{2}, \mathbf{r}_{3}, \mathbf{r}_{4}, \ldots, \mathbf{r}_{N}, t\right) .
$$

The following steps are now made to obtain from Eq. (7) a closed equation of motion for the total-correlation function $h \equiv g-1$, the Fourier transform of which is essentially the structure factor that is measured in a light scattering experiment:

(i) Since the total-correlation function $h$ vanishes at infinite separation between two particles, its asymptotic behavior at large distances is obtained by linearization of Eq. (7) with respect to $h$, as far as the long-ranged contributions of $h$ are concerned. It will be shown later that linearization is allowed only when the critical point is not approached very closely, and complies with a mean field approximation.

(ii) The classic superposition approximation for the triplet correlation function,

$$
g_{3}\left(\mathbf{r}_{1}, \mathbf{r}_{2}, \mathbf{r}_{3}, t\right)=g\left(\mathbf{r}_{1}-\mathbf{r}_{3}, t\right) g\left(\mathbf{r}_{3}-\mathbf{r}_{2}, t\right) g\left(\mathbf{r}_{2}-\mathbf{r}_{1}, t\right),
$$

is a very bad approximation close to the critical point (this closure leads to a finite correlation length at the critical point [48,32]. According to Eq. (8) for the indirect force, a closure relation is needed only for distances $r^{\prime}<R_{V}$, with $R_{V}$ the range of the pair-interaction potential, while $r \gg R_{V}$. Hence, the only configurations for which a closure relation is needed are those where the two particles at $\mathbf{r}_{1}$ and $\mathbf{r}_{3}$ are in each other's vicinity, while the third particle at $\mathbf{r}_{2}$ is far away from these two particles. The effect of the presence of the third particle is that it enhances the local density around the two neighboring particles. This density enhancement is equal to $\rho h\left(\left(\mathbf{r}_{1}+\mathbf{r}_{3}\right) / 2-\mathbf{r}_{2}, t\right)$. The pair-correlation function $g\left(\mathbf{r}=\mathbf{r}_{1}\right.$ $\left.-\mathbf{r}_{3}, t\right)$ in the classical superposition approximation (10) is now simply replaced by the pair-correlation function evaluated at the local density $\rho\left[1+h\left(\left(\mathbf{r}_{1}+\mathbf{r}_{3}\right) / 2-\mathbf{r}_{2}, t\right)\right]$. To leading order in $h$ we thus have

$$
\begin{aligned}
g_{3}\left(\mathbf{r}_{1}, \mathbf{r}_{2}, \mathbf{r}_{3}, t\right) \\
=[ \\
\quad\left[g\left(\mathbf{r}_{1}-\mathbf{r}_{3}, t\right)+\frac{d g\left(\mathbf{r}_{1}-\mathbf{r}_{3}, t\right)}{d \rho} \rho h\left(\left(\mathbf{r}_{1}+\mathbf{r}_{3}\right) / 2-\mathbf{r}_{2}, t\right)\right] \\
\quad \times g\left(\mathbf{r}_{3}-\mathbf{r}_{2}, t\right) g\left(\mathbf{r}_{2}-\mathbf{r}_{1}, t\right) .
\end{aligned}
$$

The pair-correlation functions here comply with the number density $\rho$. This "improved superposition approximation" leads to the correct (mean field type) divergence of the correlation length on approach to the critical point.

(iii) The third approximation concerns the neglect of shear-induced distortions of short-ranged correlations. First notice that in Eq. (8) we need only $g\left(\mathbf{r}^{\prime}=\mathbf{r}_{1}-\mathbf{r}_{3}, t\right)$ from the closure in Eq. (11) for distances between particles 1 and 3 smaller than the range $R_{V}$ of the pair-interaction potential. The reason for the neglect of the shear-rate dependence of $g\left(\mathbf{r}^{\prime}\right)$ is that long-ranged, slowly fluctuating critical microstructural order is distorted much more easily than shortranged, relatively fast fluctuating structural order. This can be quantified as follows. The distortion of correlations that extend over distances of at most $R_{V}$ is measured by the socalled bare Peclet number

$$
\mathrm{Pe}^{0}=\frac{\dot{\gamma} R_{V}^{2}}{2 D_{0}}
$$

As will be shown later, the distortion of near-critical microstructural order is measured by the dressed Peclet number

$$
\lambda=\frac{\dot{\gamma} \xi^{2}}{2 D^{e f f}},
$$

where $\xi$ is the correlation length of the unsheared, quiescent suspension, and $D^{\text {eff }}$ is an effective diffusion coefficient that describes the relaxation of long-wavelength density fluctuations (a precise definition will be given later). Since $\xi \gg R_{V}$ and $D^{e f f} \ll D_{0}$, due to "critical slowing down," the dressed Peclet number is usually very large for shear rates where the bare Peclet number is still small. We shall therefore write

$$
g\left(\mathbf{r}^{\prime}=\mathbf{r}_{1}-\mathbf{r}_{3}, t\right)=g^{e q}\left(r^{\prime}\right) \text { for } r^{\prime}<R_{V},
$$

where $g^{e q}$ is the equilibrium pair-correlation function, that is, the correlation function of the quiescent, unsheared system.

(iv) Since the interest here is in the long-wavelength critical microstructure, convolution type integrals that are encountered can be gradient expanded up to leading order.

An analysis of the equation of motion (7) invoking these three approximations leads to the following equation of motion for the total correlation function:

$$
\begin{aligned}
\frac{\partial h(\mathbf{r}, t)}{\partial t}= & 2 \beta D_{0} \nabla \cdot\left[\frac{d \Pi}{d \rho} \nabla h(\mathbf{r}, t)-\Sigma \nabla \nabla^{2} h(\mathbf{r}, t)\right] \\
& -\dot{\gamma} \boldsymbol{\nabla} \cdot[\hat{\boldsymbol{\Gamma}} \cdot \mathbf{r} h(\mathbf{r}, t)],
\end{aligned}
$$

where

$$
\Pi=\beta^{-1} \rho-\frac{2 \pi}{3} \rho^{2} \int_{0}^{\infty} d r^{\prime} r^{\prime 3} \frac{d V\left(r^{\prime}\right)}{d r^{\prime}} g^{e q}\left(r^{\prime}\right)
$$

is the osmotic pressure, and

$$
\Sigma=\frac{2 \pi}{15} \rho \int_{0}^{\infty} d r^{\prime} r^{\prime} \frac{d V\left(r^{\prime}\right)}{d r^{\prime}}\left[g^{e q}\left(r^{\prime}\right)+\frac{1}{8} \rho \frac{d g^{e q}\left(r^{\prime}\right)}{d \rho}\right]
$$

is the Cahn-Hilliard square gradient coefficient. Fourier transformation leads to the equation of motion for the structure factor, 


$$
\frac{\partial S(\mathbf{k}, t)}{\partial t}=\dot{\gamma} k_{1} \frac{\partial S(\mathbf{k}, t)}{\partial k_{2}}-2 D^{e f f}(k) k^{2}\left[S(\mathbf{k}, t)-S^{e q}(k)\right],
$$

where $k_{j}$ is the $j$ th component of the wave vector $\mathbf{k}$, and $S^{e q}$ is the Ornstein-Zernike structure factor,

$$
S^{e q}(k)=\frac{1}{\beta \Sigma} \frac{1}{\xi^{-2}+k^{2}},
$$

where $\xi$ is the correlation length of the non sheared system,

$$
\xi=\sqrt{\Sigma / \frac{d \Pi}{d \rho}} .
$$

Furthermore, the wave-vector-dependent effective diffusion coefficient is defined as

$$
D^{e f f}(k)=D_{0} \beta\left[\frac{d \Pi}{d \rho}+\Sigma k^{2}\right]=D_{0} \beta \Sigma\left[\xi^{-2}+k^{2}\right] .
$$

Since $\beta d \Pi / d \rho \rightarrow 0$ on approach to the critical point, the correlation length diverges as it should, while $D^{e f f}(k=0) \rightarrow 0$, implying that concentration fluctuations of long wavelength become very slow, which is commonly referred to critical slowing down.

The neglect of quadratic terms in $h$ in the equation of motion (15) [approximation (i)] is only allowed when typical values of $h^{2}$ at large distances are much smaller than $h \beta d \Pi / d \rho$. This is violated very close to the critical point. Linearization with respect to $h$ can thus be regarded as a mean field approximation.

In order to quantify the response to (oscillatory) shear flow, Eq. (18) is written in dimensionless form. To this end, the dimensionless time and wave vector

$$
\begin{gathered}
\tau=\frac{2 D^{e f f}}{\xi^{2}} t, \\
\mathbf{K}=\mathbf{k} \xi
\end{gathered}
$$

are introduced. Time is thus expressed in units of the time that a colloid takes to diffuse over a distance equal to the correlation length, and the wave vector in units of the inverse correlation length. For stationary shear flow, the equation of motion (18) transforms to

$$
0=\lambda K_{1} \frac{\partial S(\mathbf{K})}{\partial K_{2}}-K^{2}\left(1+K^{2}\right)\left[S(\mathbf{K})-S^{e q}(K)\right],
$$

where $\lambda$ is the dressed Peclet number defined in Eq. (13) with,

$$
D^{e f f}=D^{e f f}(k=0)=D_{0} \beta \Sigma \xi^{-2},
$$

where in the last equality Eq. (21) has been used. For oscillatory shear flow [see Eq. (3)] it is found that $\frac{\partial S(\mathbf{K}, \tau)}{\partial \tau}=\lambda_{0} \cos \{\Omega \tau\} K_{1} \frac{\partial S(\mathbf{K})}{\partial K_{2}}-K^{2}\left(1+K^{2}\right)\left[S(\mathbf{K})-S^{e q}(K)\right]$,

where the Peclet number $\lambda_{0}$ for oscillatory shear flow is similarly defined as in Eq. (13) for stationary flow,

$$
\lambda_{0}=\frac{\dot{\gamma}_{0} \xi^{2}}{2 D^{e f f}},
$$

and where $\Omega$ is the Deborah number

$$
\Omega=\frac{\omega \xi^{2}}{2 D^{e f f}} .
$$

Equations (23) and (25) can be solved analytically (see Refs. [32] and [41]). Since more general equations of motion will be solved in the next section, we shall not display these results here.

Since in Eqs. (23) and (25) the shear rate is always multiplied by $K_{1}$, these equations predict that there is no effect of shear flow in directions perpendicular to the flow direction. As will be discussed later, this is not confirmed by experiments. The experiments reveal large distortions also in directions perpendicular to the flow. The reason for this discrepancy is discussed in the subsequent section.

Note that the Peclet number (13) and the Deborah number (27) can also be written as

$$
\lambda=\frac{\dot{\gamma} \xi^{4}}{2 D_{0} \beta \Sigma}, \quad \Omega=\frac{\omega \xi^{4}}{2 D_{0} \beta \Sigma} .
$$

Hence, both $\lambda$ and $\Omega$ are $\sim \xi^{4}$, with the same proportionality constant. This dependence on the correlation length can be verified experimentally. The large values of $\lambda$ and $\Omega$ for relatively small values of $\dot{\gamma}$ and $\omega$, respectively, quantifies the large susceptibility of near-critical systems.

\section{THE EXTENDED THEORY: THE ROLE OF DISTORTIONS OF SHORT-RANGED CORRELATIONS}

The approximation (iii) discussed in the previous section states that short-ranged correlations are relatively little affected by flow as compared to long-ranged correlations. We shall show in the present section that this seemingly straightforward approximation is not correct, despite the fact that the Peclet numbers (12) and (13), which measure the distortion of short- and long-ranged correlations, respectively, are orders of magnitude different.

Instead of completely neglecting short-ranged distortions, we shall keep leading order deviations from equilibrium structure, that is, instead of Eq. (14), we shall expand the correlation function to first order in the bare Peclet number as

$$
\begin{aligned}
g\left(\mathbf{r}^{\prime}, t\right)= & g^{e q}\left(r^{\prime}\right)\left\{1+\left|\mathrm{Pe}^{0}\right| f_{0}\left(r^{\prime}\right)-\mathrm{Pe}^{0} \frac{\mathbf{r}^{\prime} \cdot \hat{\mathbf{E}} \cdot \mathbf{r}^{\prime}}{r^{\prime 2}} f_{1}\left(r^{\prime}\right)\right\} \\
& \text { for } r^{\prime}<R_{V},
\end{aligned}
$$

where (the superscript $T$ stands for transposition) 


$$
\hat{\mathbf{E}}=\frac{1}{2}\left[\hat{\boldsymbol{\Gamma}}+\hat{\boldsymbol{\Gamma}}^{T}\right]
$$

is the symmetric part of the dimensionless velocity gradient tensor. Furthermore, the functions $f_{0}$ and $f_{1}$ describe the isotropic and anisotropic responses of the short-ranged part of the pair-correlation function to shear flow, respectively. Here, $\mathrm{Pe}^{0}$ is the bare Peclet number in Eq. (12), where $\dot{\gamma}$ is either a constant for stationary flow or given by Eq. (3) for oscillatory flow.

When $g$ for short distances is an analytic function of the shear rate, the leading order isotropic distortion is quadratic in the Peclet number for symmetry reasons. That is, the isotropic contribution in Eq. (29) is absent when the shortranged part of $g$ can be expanded in a converging power series of $\dot{\gamma}$. As we shall see later, we do need an isotropic distortion that is linear in the shear rate to explain experimental data. For symmetry reasons, such linear isotropic distortion can only be proportional to the absolute value of the shear rate. This implies that the pair-correlation function for short distances is a nonanalytic function of the shear rate. The probable reason for this is as follows. In the equation of motion for $g$, hydrodynamic interactions give rise to terms proportional to integrals containing triplet and four-point pair-correlation functions. These integrals extend over the entire space, and thus probe the long-ranged behavior of $g$. Since close to the critical point these long-range correlations are significant, it might be that these terms give rise to the nonanalytic behavior of $g$ for small distances. This is how long-ranged correlations could give rise to a nonanalytic response of short-ranged correlations.

\section{A. Stationary shear flow}

Repeating the analysis discussed in the previous section, using Eq. (29) instead of assumption (iii), Eq. (23) for stationary shear flow is found to extend to

$$
\begin{aligned}
0= & \lambda K_{1} \frac{\partial S(\mathbf{K})}{\partial K_{2}}-K^{2}\left(1+\epsilon+K^{2}\right) S(\mathbf{K})+K^{2}\left(1+K^{2}\right) S^{e q}(K) \\
& +\alpha K_{1} K_{2}[S(\mathbf{K}-1)]
\end{aligned}
$$

There are two new parameters $\epsilon$ and $\alpha$ which are defined as

$$
\epsilon=\frac{|\dot{\gamma}|\left(R_{V} \xi\right)^{2}}{2 D_{0} \Sigma} F_{0}
$$

with

$$
\begin{gathered}
F_{0}=\frac{4 \pi}{3} \rho \int_{0}^{\infty} d r r^{3} \frac{d V(r)}{d r}\left\{g^{e q}(r) f_{0}(r)+\frac{1}{2} \bar{\rho} \frac{d\left[g^{e q}(r) f_{0}(r)\right]}{d \bar{\rho}}\right\}, \\
\alpha=\frac{\dot{\gamma}\left(R_{V} \xi\right)^{2}}{2 D_{0} \Sigma} F_{1}
\end{gathered}
$$

with

$$
F_{1}=\frac{8 \pi}{15} \rho \int_{0}^{\infty} d r r^{3} \frac{d V(r)}{d r}\left\{g^{e q}(r) f_{1}(r)+\frac{1}{2} \bar{\rho} \frac{d\left[g^{e q}(r) f_{1}(r)\right]}{d \bar{\rho}}\right\}
$$

The dimensionless parameter $\epsilon$ measures the susceptibility of isotropic short-ranged distortions, and $\alpha$ of the anisotropic distortions.

The solution of Eq. (31) reads

$$
\begin{aligned}
S(\mathbf{K})= & \frac{1}{\lambda K_{1}} \int_{K_{2}}^{ \pm \infty} d X\left\{( K ^ { 2 } - K _ { 2 } ^ { 2 } + X ^ { 2 } ) \left(1+K^{2}-K_{2}^{2}\right.\right. \\
& \left.\left.+X^{2}\right) S^{e q}\left(\sqrt{K_{1}^{2}+X^{2}+K_{3}^{2}}\right)-\alpha K_{1} X\right\} \exp \left\{-F(\mathbf{K} \mid X) / \lambda K_{1}\right\},
\end{aligned}
$$

with

$$
\begin{aligned}
F(\mathbf{K} \mid X)= & \left(X-K_{2}\right)\left(K^{2}-K_{2}^{2}\right)\left(1+\epsilon+K^{2}-K_{2}^{2}\right)+\frac{1}{3}\left(X^{3}-K_{2}^{3}\right) \\
& \times\left(1+\epsilon+2 K^{2}-2 K_{2}^{2}\right)+\frac{1}{5}\left(X^{5}-K_{2}^{5}\right)-\alpha K_{1}\left(X^{2}-K_{2}^{2}\right) .
\end{aligned}
$$

The upper integration limit in Eq. (33) is equal to $+\infty$ when $\lambda K_{1}>0$ and equal to $-\infty$ when $\lambda K_{1}<0$. For $\lambda \rightarrow 0$, the function that multiplies $S^{e q}$ in the above equation becomes a $\delta$-function distribution in $X$, so that $S$ becomes equal $S^{e q}$ without shear flow, as it should.

Contrary to the case where short-ranged distortions are neglected, one now obtains a finite distortion in directions perpendicular to the flow direction where $K_{1}=0$. In these directions, according to Eq. (31), the structure factor attains an Ornstein-Zernike-like form,

$$
S(\mathbf{K})=\frac{1}{\beta \Sigma} \frac{1}{\left(\xi^{e f f}\right)^{-2}+k^{2}} \quad\left(K_{1}=0\right),
$$

where the "effective," shear-rate-dependent correlation length is equal to

$$
\xi^{e f f}=\xi / \sqrt{1+\epsilon} .
$$

The interpretation of this result is that the location of the critical point is shifted on applying shear flow. The accompanied shear-induced change of the correlation length is thus equal to (note that $\epsilon>0$ )

$$
\frac{\Delta \xi}{\xi} \equiv \frac{\xi^{e f f}-\xi}{\xi}=\frac{1}{\sqrt{1+\epsilon}}-1<0 .
$$

This shear-induced decrease of the correlation length can be interpreted as being a consequence of a shear-induced shift of the critical point. That the shear-induced displacement of the gas-liquid spinodal (including the critical point) is indeed related to shear distortion of short-ranged correlations has been shown in [42].

\section{B. Oscillatory shear flow}

Including short-ranged distortions, the equation of motion Eq. (25) for oscillatory shear flow extends to 


$$
\begin{aligned}
\frac{\partial S(\mathbf{K}, \tau)}{\partial \tau}= & \lambda_{0} \cos \{\Omega \tau\} K_{1} \frac{\partial S(\mathbf{K}, \tau)}{\partial K_{2}} \\
& -K^{2}\left(1+\left|\epsilon_{0} \cos \{\Omega \tau\}\right|+K^{2}\right) S(\mathbf{K}, \tau) \\
& -K^{2}\left(1+K^{2}\right) S^{e q}(K)+\alpha_{0} K_{1} K_{2}[S(\mathbf{K}, \tau)-1]
\end{aligned}
$$

where the dimensionless numbers $\epsilon_{0}$ and $\alpha_{0}$ are defined as

$$
\begin{gathered}
\epsilon_{0}=\frac{\left|\dot{\gamma}_{0}\right|\left(R_{V} \xi\right)^{2}}{2 D_{0} \Sigma} F_{0}, \\
\alpha=\frac{\dot{\gamma}_{0}\left(R_{V} \xi\right)^{2}}{2 D_{0} \Sigma} F_{1},
\end{gathered}
$$

where $F_{0}$ and $F_{1}$ are given in Eq. (32).

The solution of this equation of motion reads

$$
\begin{aligned}
S(\mathbf{K}, \tau)= & \int_{-\infty}^{\tau} d \tau^{\prime}\left\{G^{2}\left(\tau^{\prime}\right)\left[1+G^{2}\left(\tau^{\prime}\right)\right] S^{e q}\left(G\left(\tau^{\prime}\right)\right)\right. \\
& \left.-\alpha_{0} \cos \left\{\Omega \tau^{\prime}\right\} G_{1}\left(\tau^{\prime}\right) G_{2}\left(\tau^{\prime}\right)\right\} \exp \left\{-H\left(\tau, \tau^{\prime}\right)\right\},
\end{aligned}
$$

where

$$
\begin{aligned}
H\left(\tau, \tau^{\prime}\right)= & \int_{\tau^{\prime}}^{\tau} d \tau^{\prime \prime}\left\{G^{2}\left(\tau^{\prime \prime}\right)\left[1+\left|\epsilon_{0} \cos \left(\Omega \tau^{\prime \prime}\right)\right|+G^{2}\left(\tau^{\prime \prime}\right)\right]\right. \\
& \left.-\alpha_{0} \cos (\Omega \tau) G_{1}\left(\tau^{\prime \prime}\right) G_{2}\left(\tau^{\prime \prime}\right)\right\}
\end{aligned}
$$

and

$$
\mathbf{G}\left(\tau^{\prime \prime}\right)=\left(K_{1}, K_{2}+\frac{\lambda_{0}}{\Omega} K_{1} \sin \{\Omega \tau\}-\frac{\lambda_{0}}{\Omega} K_{1} \sin \left\{\Omega \tau^{\prime \prime}\right\}, K_{3}\right) .
$$

In Eq. (40), $G_{1}\left(\tau^{\prime}\right)$ and $G_{2}\left(\tau^{\prime}\right)$ are the first and second components of $\mathbf{G}\left(\tau^{\prime}\right)$ in Eq. (42), respectively. Note that in directions perpendicular to the flow direction, where $K_{1}=0$, this result reduces to

$S(\mathbf{K}, \tau)=K^{2}\left(1+K^{2}\right) S^{e q}(K) \int_{-\infty}^{\tau} d \tau^{\prime} \exp \left\{-H\left(\tau, \tau^{\prime}\right)\right\}\left(K_{1}=0\right)$,

with

$$
H\left(\tau, \tau^{\prime}\right)=K^{2}\left(1+K^{2}\right)\left(\tau-\tau^{\prime}\right)+K^{2} \int_{\tau^{\prime}}^{\tau} d \tau^{\prime \prime}\left|\epsilon_{0} \cos \left(\Omega \tau^{\prime \prime}\right)\right| .
$$

As for stationary shear flow, the structure factor deformation in directions perpendicular to the flow direction is nonzero due to the effect of the flow on short-ranged correlations.

\section{EXPERIMENTS}

\section{A. System and light scattering setup}

The system used in this study consists of colloidal silica spheres (102 nm diameter) grafted with stearyl alcohol and dissolved in cyclohexane. These spheres behave as almost perfect hard spheres. Polydimethylsiloxane (PDMS, Jansen) with a molecular weight of $206 \mathrm{~kg} / \mathrm{mol}$ and a radius of gyration of $23 \mathrm{~nm}$ was added to induce depletion attractions between the colloidal particles, giving rise to a gas-liquid phase transition. The critical point of the dispersion was found at a colloid volume fraction of $19 \%$ and a polymer concentration of $6.5 \mathrm{mg} / \mathrm{ml}$, by locating the point on the binodal where, after phase separation, the volumes of the two phases are equal. The distance to the critical point can be tuned by gently evaporating from or adding solvent to a dispersion with the critical composition ratio of silica to polymer. For more details see Ref. [49].

Small-angle, time-resolved light scattering experiments are performed using a home-built optical couette shear cell, where the radius of the inner cylinder is $21 \mathrm{~mm}$ and with a gap width of $2.47 \mathrm{~mm}$. For oscillatory experiments, the inner cylinder oscillates with angular amplitudes between $10^{\circ}$ and $130^{\circ}$, while frequencies are varied from 0.025 to $0.1 \mathrm{~Hz}$. The shear cell is placed in a cylindrical toluene bath with the second gap of the cell in the center of the bath. Scattered light originating from the first gap is blocked by means of a pinhole that is positioned within the hollow inner cylinder. The inner cylinder is filled with toluene. A $5.0 \mathrm{~mW} \mathrm{He}-\mathrm{Ne}$ laser (Melles-Griott) with a wavelength of $632.8 \mathrm{~nm}$ was used as a light source. The laser beam is directed along the gradient direction, so that the flow-vorticity plane is probed in our experiments. The scattered intensity is projected on a screen. A hole in the center of this screen prevents detection of stray light from the laser beam. The size of this hole corresponds to a scattering angular range $0-1.4^{\circ}$ and a wave vector range of $0-0.24 \mu \mathrm{m}^{-1}$. Images of the screen were taken in backscattering mode with a peltier cooled 12-bit charge-coupled device (CCD) camera, with $582 \times 782$ pixels (Princeton Instruments, microMAX). The maximum scattering angle was $7.7^{\circ}$, which corresponds to a wave vector of $1.3 \mu \mathrm{m}^{-1}$. The deformation of the scattering pattern due to the cylindrically shaped toluene bath was corrected for. A Hameg HM8131-2 function generator was used to control the oscillatory motion of the motor. The amplitude of the oscillation was measured at the axis of the couette cell with an angle recorder (TWK-elektronik), which was connected to a LeCroy oscilloscope. A measurement consists of a series of 128 or 256 images (depending on the period of oscillation) which were taken at time intervals between 0.25 and $0.5 \mathrm{~s}$. The start of a series of images was triggered by the Hameg function generator, such that each set of images starts at an instant of time where the shear rate is zero. For a typical experiment ten of such time series were averaged. The experiment was controlled by a home-written LABVIEW program. A schematic overview of the setup is given in Fig. 1.

\section{B. Relation between the scattered intensity and the structure factor}

The high turbidity of the near-critical samples must be accounted for on relating measured intensities to the structure factor. The turbidity not only depends on the distance to the critical point but is also a function of the shear rate [29], since it is related to the structure factor as 


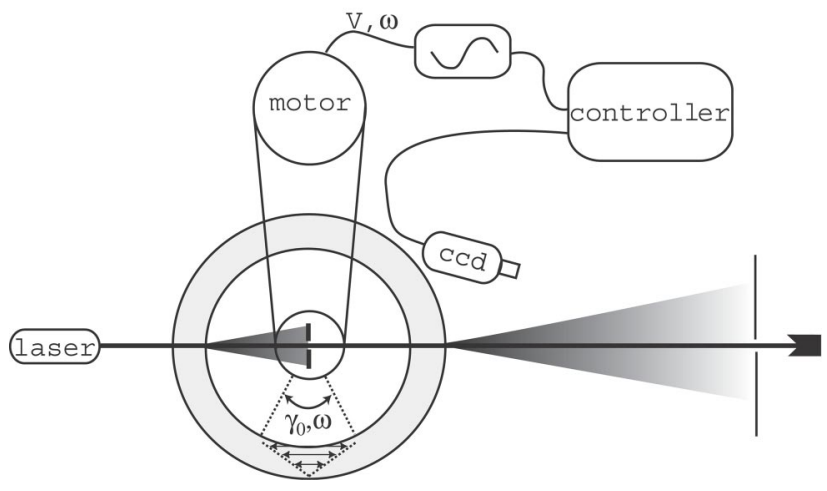

FIG. 1. Schematic overview of the experimental setup for smallangle light scattering under (oscillatory) shear flow.

$$
\tau=\frac{C_{\tau}}{k_{0}^{2}} \int_{0}^{2 \pi} d \phi \int_{0}^{2 k_{0}} d k k P(k) S(\mathbf{k}) .
$$

Here $C_{\tau}$ is an experimental constant determined by the volume and dielectric constant of the colloidal particles, $P(k)$ is the form factor of the colloidal spheres (see [33]), and $k_{0}$ $=2 \pi / \lambda$ is the wave vector of the incident light, with $\lambda$ the wavelength of the laser light within the dispersion $[29,45]$. The structure factor is related to the measured intensity as

$$
S(\mathbf{K})=\frac{I(\mathbf{K})-I_{b a c k}(\mathbf{K})}{A} .
$$

Here, the "susceptibility" $A$ is the proportionality constant between experimental intensities and the structure factor. Furthermore, $I_{\text {back }}(\mathbf{K})$ is the background intensity, which is the intensity as measured from the couette cell filled with just solvent. The background intensity $I_{b a c k}$ arises from stray light and reading noise of the CCD camera. The turbidity dependence of the susceptibility $A$ can be accounted for explicitly by writing it as

$$
A(\xi, \dot{\gamma})=C_{\text {expt }} \exp \{\tau(\xi, \dot{\gamma}) l\}
$$

with $C_{\text {expt }}$ an experimental constant that is independent of the turbidity, and $l$ the optical path length, which is equal to two times the gap width of the couette cell. The dependence of the turbidity on the distance from the critical point (measured by the correlation length $\xi$ without shear flow) and the shear rate is $\dot{\gamma}$ denoted here explicitly. The relation between the turbidity and the structure factor thus leaves a single adjustable parameter $C_{\text {expt }}$ to relate intensities to the structure factor.

\section{The structure factor without shear flow}

An experimental equilibrium Ornstein-Zernike structurefactor is plotted in Fig. 2. The equilibrium correlation length $\xi$ is obtained from this structure factor (after angular averaging of the two-dimensional scattering pattern) by an Ornstein-Zernike fit, that is, a linear fit of $1 / S^{e q}(k)$ vs $k^{2}$. According to Eq. (19), the square root of the slope divided by the intercept of such a plot gives the correlation length. The corresponding Ornstein-Zernike plots are given in Fig. 3

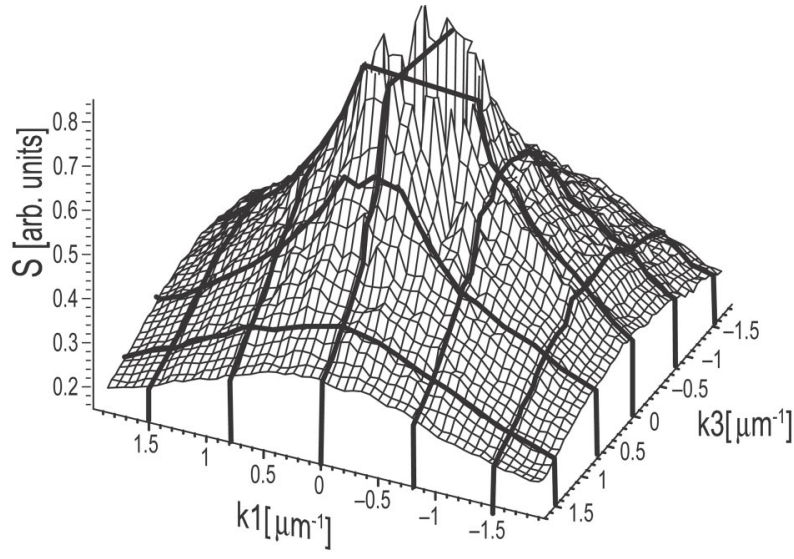

FIG. 2. The equilibrium Ornstein-Zernike structure factor, corresponding to a correlation length of $\xi=650 \mathrm{~nm}$. The thick lines indicate typical cross sections that are used for data analysis of sheared systems.

for equilibrium suspensions, which are used in the shear experiments later on.

\section{Structure factor under stationary shear flow}

We measured the shear-induced distortion of the structure factor for shear rates ranging from $\dot{\gamma}=0$ to $33.5 \mathrm{~s}^{-1}$, for correlation lengths in the range of 300 to $1500 \mathrm{~nm}$. Experimental data for the distortion

$$
\Delta S(\mathbf{K}) \equiv \frac{\beta \Sigma}{\xi^{2}}\left\{S_{e q}(K)-S_{\dot{\gamma}}(\mathbf{K})\right\}
$$

are compared to the experimental predictions in Eqs. (33) and (34). Experimental data for $\Delta S$ will also be compared to the theory where short-ranged distortions of the paircorrelation function are not included. For convenience of data analysis we extract data from ten cross sections from each scattering pattern: five cross sections at fixed values of $K_{1}$ as functions of $K_{3}$, and five cross sections at fixed $K_{3}$ as functions of $K_{1}$. These cross sections are indicated by the thick lines in Fig. 2. In accordance with theory, the scattered intensity for cross sections is found to be invariant under

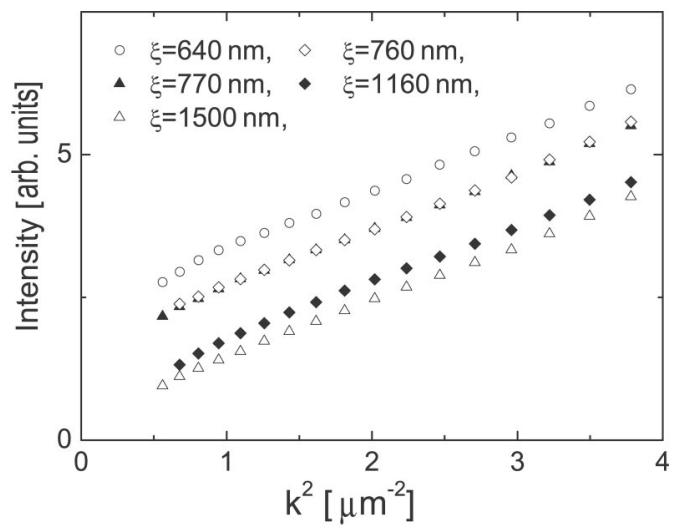

FIG. 3. Ornstein-Zernike plots from which equilibrium correlation lengths are determined, as indicated in the figure. 

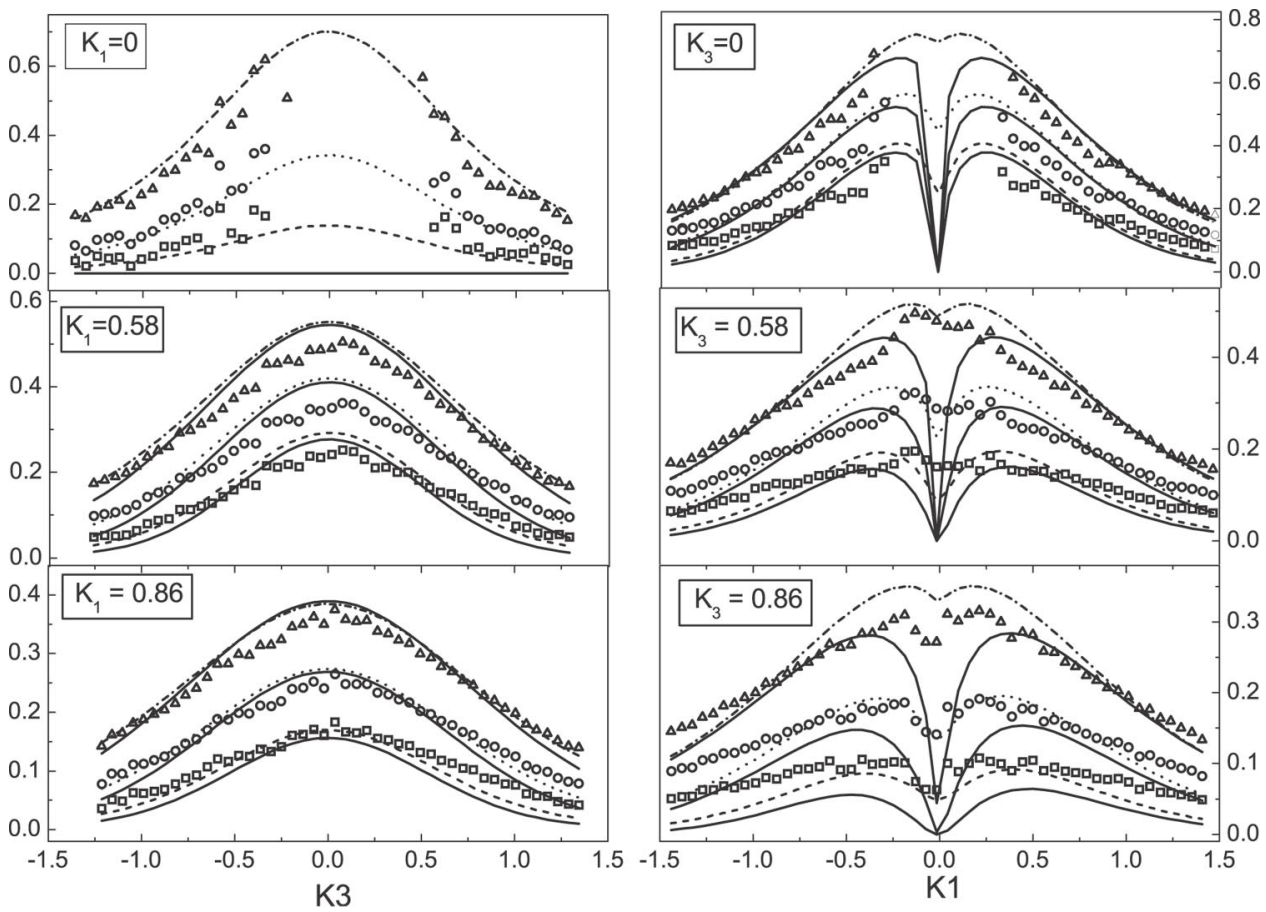

FIG. 4. Typical fitting results of the shear-induced distortion of the structure factor $\Delta S$ for a correlation length of $770 \mathrm{~nm}$ and three shear rates: $33.5 \mathrm{~s}^{-1}(\triangle), 7.45 \mathrm{~s}^{-1}$ (O), and $2.3 \mathrm{~s}^{-1}(\square)$. Points are experimental data, solid lines are fits to the theory where distortions of short-ranged correlations are neglected, while the dash-dotted, dotted, and dashed lines are fits to the theory with the inclusion of shear-induced distortions of shortranged correlations. The values for the wave vectors indicated in the figures are the wave vectors where cross sections are taken, as indicated by the thick lines in Fig. 2. Note the difference in scale of the vertical axis for the different cross sections. coordinate inversion to within experimental error. This results in six independent scattering curves per scattering pattern, that is, for each correlation length and each shear rate. These six scattering curves are fitted simultaneously for all correlation lengths and shear rates together to the theoretical predictions. There are only three shear-rate-independent fit parameters: $\lambda / \dot{\gamma}, \alpha / \dot{\gamma}$, and $\epsilon / \dot{\gamma}$. The effect of the turbidity, which also depends on these three parameters, was included in the fit, using Eq. (45) to calculate the turbidity. The prefactor $C_{\text {expt }}$ in the integral in Eq. (45) constitutes a fourth fit parameter, which is independent of both the shear rate and the equilibrium correlation length. Its value was found to be equal to $C_{\tau} / k_{0}^{2}=0.25$.
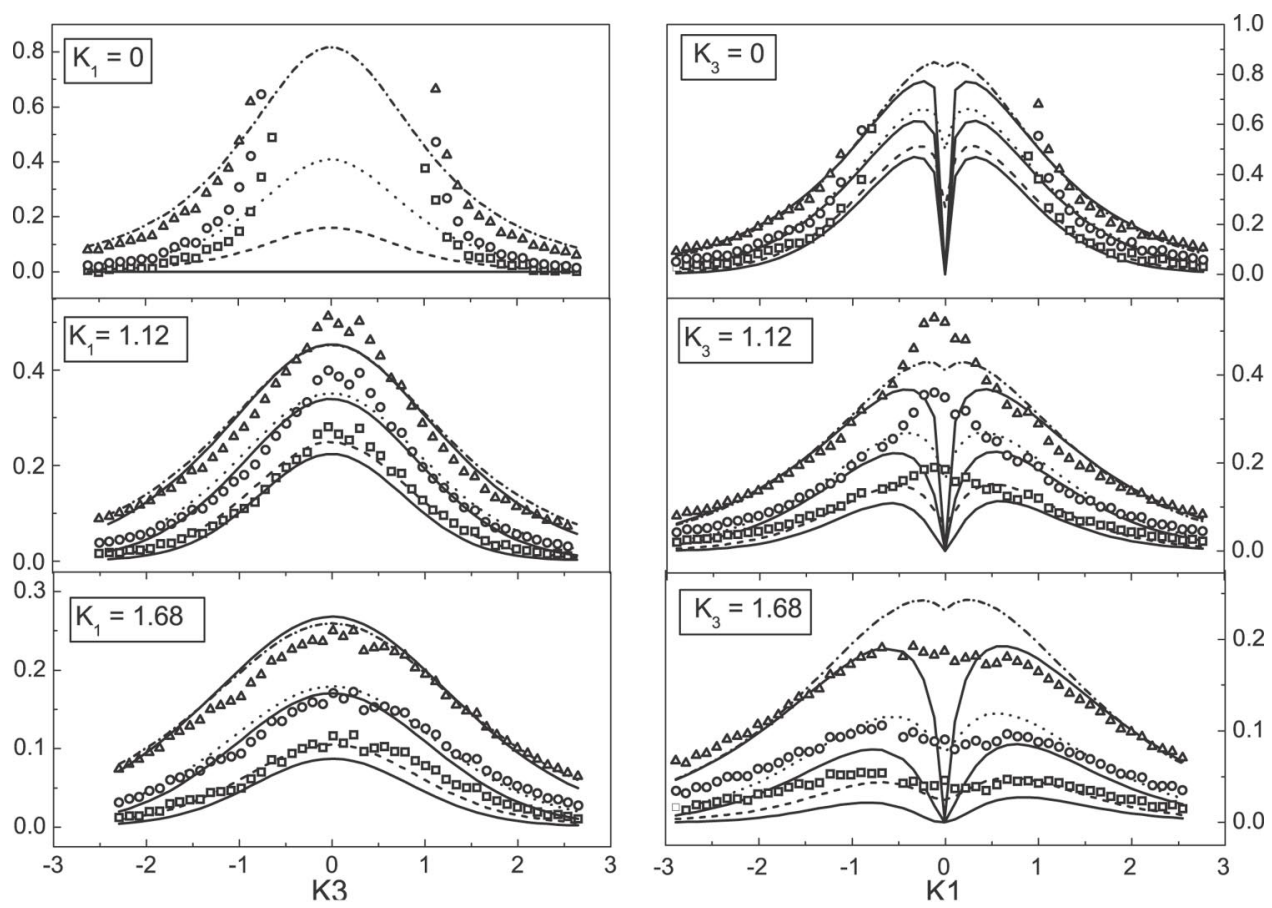

$061405-8$
Figures 4 and 5 show the fit results for the equilibrium correlation lengths 700 and $1500 \mathrm{~nm}$, respectively. The wave vector is always expressed in dimensionless units: $K=k \xi$, with $k$ the physical wave vector. The finite distortion of the structure factor in directions perpendicular to the flow direction is most clearly seen in the top left corner plots in these figures, which are cross sections where $k_{1}=0$. As discussed in Sec. II, when contributions from shear-induced distortions of short-ranged correlations are neglected, theory predicts no effect of shear flow in directions where $k_{1}=0$. Clearly, the effect of distortions of short-ranged correlations is essential to explain our experimental data on shear-induced longranged correlations. Moreover, the extended theory, where
FIG. 5. Same as in Fig. 4, now for a correlation length of $1500 \mathrm{~nm}$ and for the three shear rates $\dot{\gamma}=20.46 \mathrm{~s}^{-1} \quad(\triangle), \quad \dot{\gamma}$ $=1.84 \mathrm{~s}^{-1}(\bigcirc)$, and $\dot{\gamma}=0.87 \mathrm{~s}^{-1}$ ( $\square$ ). 


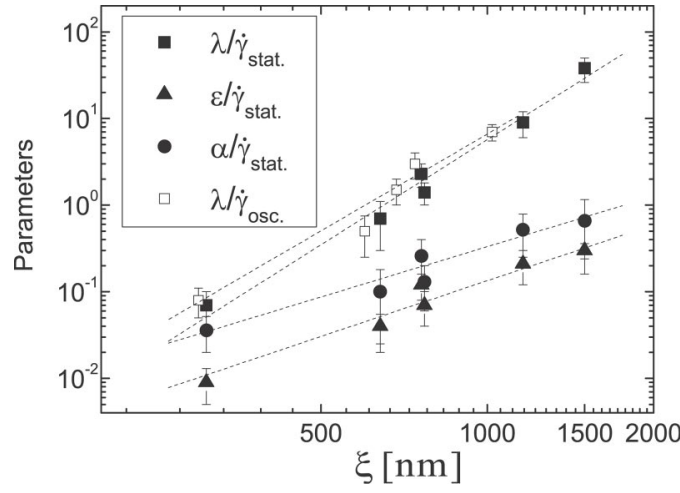

FIG. 6. The parameters $\lambda / \dot{\gamma}, \alpha / \dot{\gamma}$, and $\epsilon / \dot{\gamma}$ obtained from the fit of the scattering data under stationary shear flow as functions of the equilibrium correlation length $\xi$. For the parameter $\lambda / \dot{\gamma}$ results from oscillatory flow experiments are also shown (open symbols), which will be discussed in the subsequent section.

short-ranged distortions are accounted for, describes the experiments quantitatively correctly. In particular, the OrnsteinZernike-like wave vector dependence in directions perpendicular to the flow direction as predicted by Eqs. (35) and (36) is confirmed. The fact that the data points are somewhat above the theoretical predictions in the middle figures is due to the contribution of additional stray intensities at small angles, close to the laser beam. Note that the predicted shallow minimum in scattering curves at fixed $k_{3}$ as functions of $k_{1}$ at $k_{1}=0$ is also seen experimentally, provided $k_{3}$ is large enough in order to be able to fully neglect stray light intensity from the incident beam.

Equations (28) and (32) predict that $\lambda / \dot{\gamma} \sim \xi^{4}, \alpha / \dot{\gamma} \sim \xi^{2}$, and $\epsilon / \dot{\gamma} \sim \xi^{2}$. This is indeed what is found from the fits at different equilibrium correlation lengths, as shown in Fig. 6. A plot of $\ln \{\lambda / \dot{\gamma}\}$ against $\ln \{\xi\}$ is predicted to be a straight line with slope 4 . A similar plot for $\epsilon / \dot{\gamma}$ and $\alpha / \dot{\gamma}$ should have a slope equal to 2 . The corresponding experimental curves in Fig. 6 are in agreement with these predictions: slopes of $3.9 \pm 0.3$ for $\lambda, 1.9 \pm 0.6$ for $\alpha$, and $2.1 \pm 0.3$ for $\epsilon$ are found. From the linear fits in Fig. 6, the prefactors given in Eqs. (28) and (32) can be obtained. Using $R_{V}=148 \mathrm{~nm}$ and the

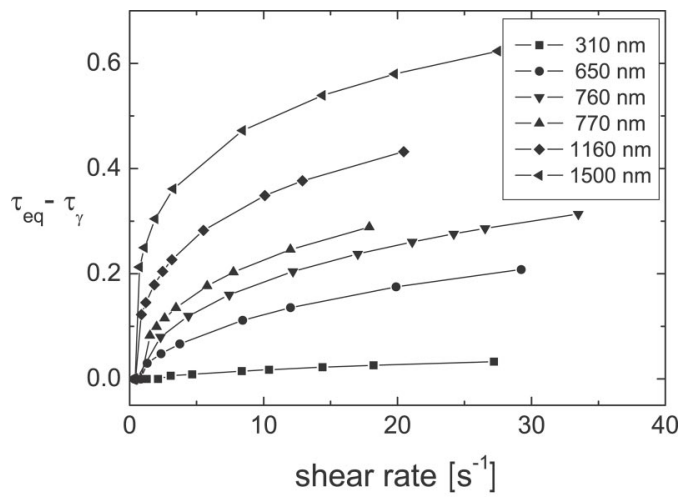

FIG. 7. The shear-rate dependence of the turbidity for four correlation lengths, where the turbidity is calculated from Eq. (45) using the fit parameters as obtained from scattering data fits. The proportionality constant in Eq. (45) used here is $C_{\tau} / k_{0}^{2}=0.25$.

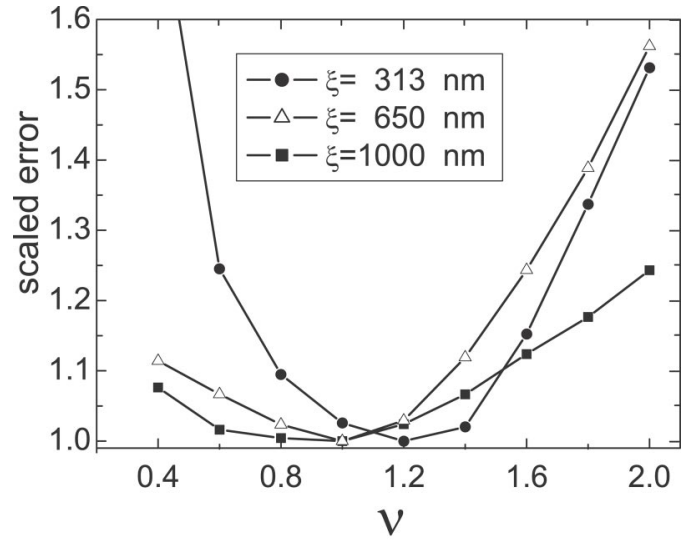

FIG. 8. The minimum standard deviation from least squares fits varying the exponent $\nu$, the shear-rate dependence of the shortrange distortion $\epsilon / \dot{\gamma}^{\nu}$, for three different correlation lengths as indicated in the figure. The error here is defined as $(1 / n) \sum_{j}\left(Y_{j}^{\text {expt }}\right.$ $\left.-Y_{j}^{\text {th }}\right)^{2}$, where $n$ is the number of data points involved in the fit, $Y_{j}^{\text {expt }}$ is the $j$ th experimental value for the structure factor, and $Y_{j}^{\text {th }}$ is the corresponding fitted, theoretical value. Errors are scaled with respect to the minimum standard deviation.

prefactor of $\lambda / \dot{\gamma}$ we find that $\beta \Sigma / R_{V}^{2}=0.71 \pm 0.08$. This value for $\beta \Sigma / R_{V}^{2}$ is of the same order as a crude, theoretical estimate $[18,45]$. The relative strength of the anisotropic and isotropic distortions of the pair-correlation function is given by the ratio of the prefactors of $\alpha$ and $\epsilon$. It turns out that the anisotropic contribution $F_{1}$ is a factor of 3.1 stronger than the isotropic contribution $F_{0}$.

Figure 7 shows the dependence of the turbidity on the shear rate, as obtained from Eq. (45) using the fit parameters as obtained from the scattering data fits. As the correlation length increases, that is, on closer approach of the critical point, the turbidity becomes a stronger function of the shear rate, and ultimately develops a nonanalytic dependence at small shear rates, in accordance with experiments on a "sticky-sphere" dispersion [29].

In Fig. 8 we also verify a posteriori that the short-ranged isotropic distortion of the pair-correlation function indeed depends linearly on the shear rate. For this verification, we performed least square fits with respect to the shear-rateindependent parameter $\epsilon / \dot{\gamma}^{\nu}$, for various values of the exponent $\nu$. Such fits correspond to expansions of the form in Eq. (29) where $\mathrm{Pe}^{0}$ is replaced by $\left(\mathrm{Pe}^{0}\right)^{\nu}$. Plotting the minimum standard deviation of these fits as a function of the value of the exponent $\nu$, it can clearly be seen that best fits are obtained for $\nu=1( \pm 0.2)$. Since the equation of motion for the pair-correlation function, even at low concentrations, is extremely complicated, it is a formidable task to actually deduce an expansion of the pair-correlation function of the form Eq. (29) from this equation of motion, especially at somewhat higher concentrations (our samples have a volume fraction of colloidal spheres of about 20\%). The experimental evidence for the expansion Eq. (29) of the short-ranged part of the pair-correlation function is indirect, since it is verified through its effect on the distortion on long-ranged correlations. Scattering data at large wave vectors on the same system would be desirable to verify Eq. (29) more directly. 


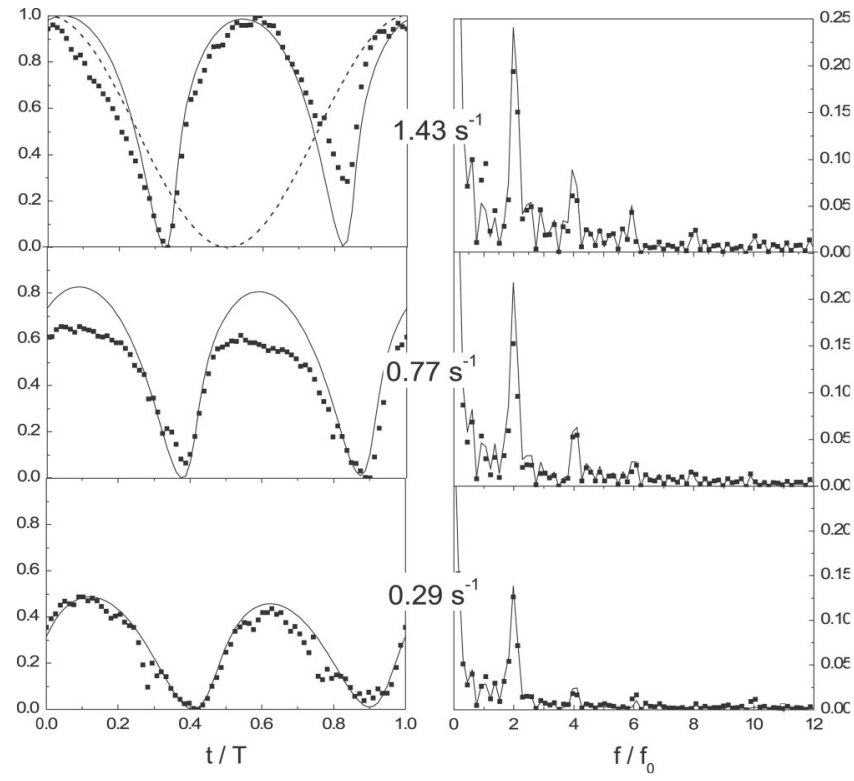

FIG. 9. The normalized responses of the structure factor $|\Delta S|$ of a near-critical colloidal dispersion with equilibrium correlation length $\xi=945 \mathrm{~nm}$ to oscillatory shear flow in the time domain (left) and frequency domain (right), for $\omega=2 \pi 0.025 \mathrm{~s}^{-1}$. The dimensionless wave vector here is equal to $\mathbf{K}=(1.4,0,0)$. The symbols represent experimental data, while solid lines indicate the theoretical prediction, using $\lambda / \dot{\gamma}=0.6$. Both experimental data and theory are normalized with respect to the response of the highest shear rate. The dashed line represents the applied field.

\section{E. Structure factor under oscillatory shear flow}

The time-dependent distortion of the structure factor due to an oscillatory shear flow is measured. The shear rate ranges from $\dot{\gamma}=0$ to $5.7 \mathrm{~s}^{-1}$, and frequencies in the range of $f=0.01$ to $0.1 \mathrm{~s}^{-1}$ are applied. Equilibrium correlation lengths vary from 300 to $945 \mathrm{~nm}$. We combined amplitudes and frequencies such that we could make series of measurements at constant shear rate $\dot{\gamma}_{0}$ in Eq. (3) and constant frequency, respectively. Typical normalized responses of the structure factor $|\Delta S|$ for the given wave vector $\mathbf{K}$ $=(1.4,0,0)$ are plotted in Fig. 9, both in the time- and frequency-domain, where the latter plots are obtained from a Fourier analysis. It is evident that the leading harmonic of the response has twice the frequency of the applied field. This is in accordance with an expansion of the structure factor in Eqs. (40)-(42) with respect to the shear rate: it turns out that in the flow-vorticity plane, where $K_{2}=0$, the leading term in the shear rate is of second order.

Typical zeroth and second order Fourier components of the structure factor are plotted in Fig. 10 for experiment and theory. The amplitude of the second harmonic can be decomposed into a real [Figs. 10(a) and 10(f)] and imaginary [Figs. $10(\mathrm{~b})$ and $10(\mathrm{~g})]$ part. These components are related to the real part (corresponding to energy dissipation) and the imaginary part (corresponding to elasticity) of the stress. As far as structure that is probed within the flow-vorticity plane is concerned, the main contribution to dissipation stems from a small range around a finite wave vector, which is oriented parallel to the shear flow. The microscopic origin for the occurrence of a maximum of the real part of the time-Fourier components of the structure factor at a finite wave vector is as follows. Relaxation rates are determined by (i) diffusion rates which scale like $\sim K^{2}$ and (ii) driving forces which scale like $\Delta S$ [see Eq. (25) with $\lambda_{0}=0$ ]. The first relaxation contribution is large at large $K$, while the second contribution is large at small $K$. This leads to a maximum of the relaxation rate at finite wave vectors. This competition of two relaxation mechanisms is responsible for a maximum in scattering pattern after cessation of shear flow [31].

The imaginary component is increasingly negative on decreasing $K_{1}$ toward 0 , meaning that the energy in an oscillatory shear flow is elastically stored in the larger structures. This is more clearly seen in Figs. 10(d) and 10(i) where instead of the imaginary component, the phase shift with respect to the applied field is plotted. Figures 10(e) and 10(j) show the time-independent component of the response, which clearly increases on approaching $K=0$. In contrast to stationary flow experiments, the absolute fits of $\Delta S$ are not quantitative. Nevertheless, theory and experiment do show the same features. The $K_{1}$ dependence of the amplitude of the response for the experimental case [Fig. 10(c)] is spread out over a larger range of $K_{1}$ values than theoretically predicted [see Fig. 10(h)]. More importantly, the experimental time-dependent components relative to the time-independent components are smaller than predicted by theory.

Because the phase shift of the structure factor response relative to the external field is a strong function of the wave vector, we relied on the fits of the $K$-dependent phase shift to determine the factor $\lambda / \dot{\gamma}$ for each of the correlation lengths. Per concentration, i.e., structure factor, the only fit parameter was $\lambda / \dot{\gamma}$. The shear-rate-dependent as well as the frequencydependent data sets were fitted simultaneously, varying this factor, since both parameters depend on that factor in the same way [see Eq. (28)]. The results are plotted in Fig. 11 for a fixed shear rate and frequency. Due to critical slowing down, the phase shift becomes more pronounced when the equilibrium correlation length increases. Furthermore, the phase shift increases dramatically with decreasing wave vector $K$ toward 0 . This is the result of the fact that relaxation times scale like $\sim K^{2}$, which signifies the larger displacements of colloidal spheres that are necessary for the relaxation of sinusoidal density profiles with longer wavelengths. In order to get a good fit we had to introduce an additional small wave-vector-independent phase shift. The origin of this additional phase shift is as yet unclear. The fits of the phase shift turned out to be insensitive to the values of $\alpha$ and $\epsilon$, which were therefore not included. The values for $\lambda / \dot{\gamma}$ vs $\xi$ obtained from the fit are plotted in Fig. 6. From the oscillatory flow experiments it is thus found that the exponent in the $\xi$ dependence of $\lambda / \dot{\gamma}$ is equal to 4 to within experimental error (the slope is $3.7 \pm 0.4$ ), in accordance with theory and the scattering experiments under stationary flow.

In Fig. 9(a), time traces of the normalized response $|\Delta S|$ are plotted for fixed frequency and wave vector, but varying the maximum shear rate. In this case the normalization is performed with respect to the maximum value of $\Delta S$ as found for the highest maximum shear rate. When increasing the shear rate for a fixed frequency, the response becomes increasingly nonlinear. The degree of nonlinearity is mea- 

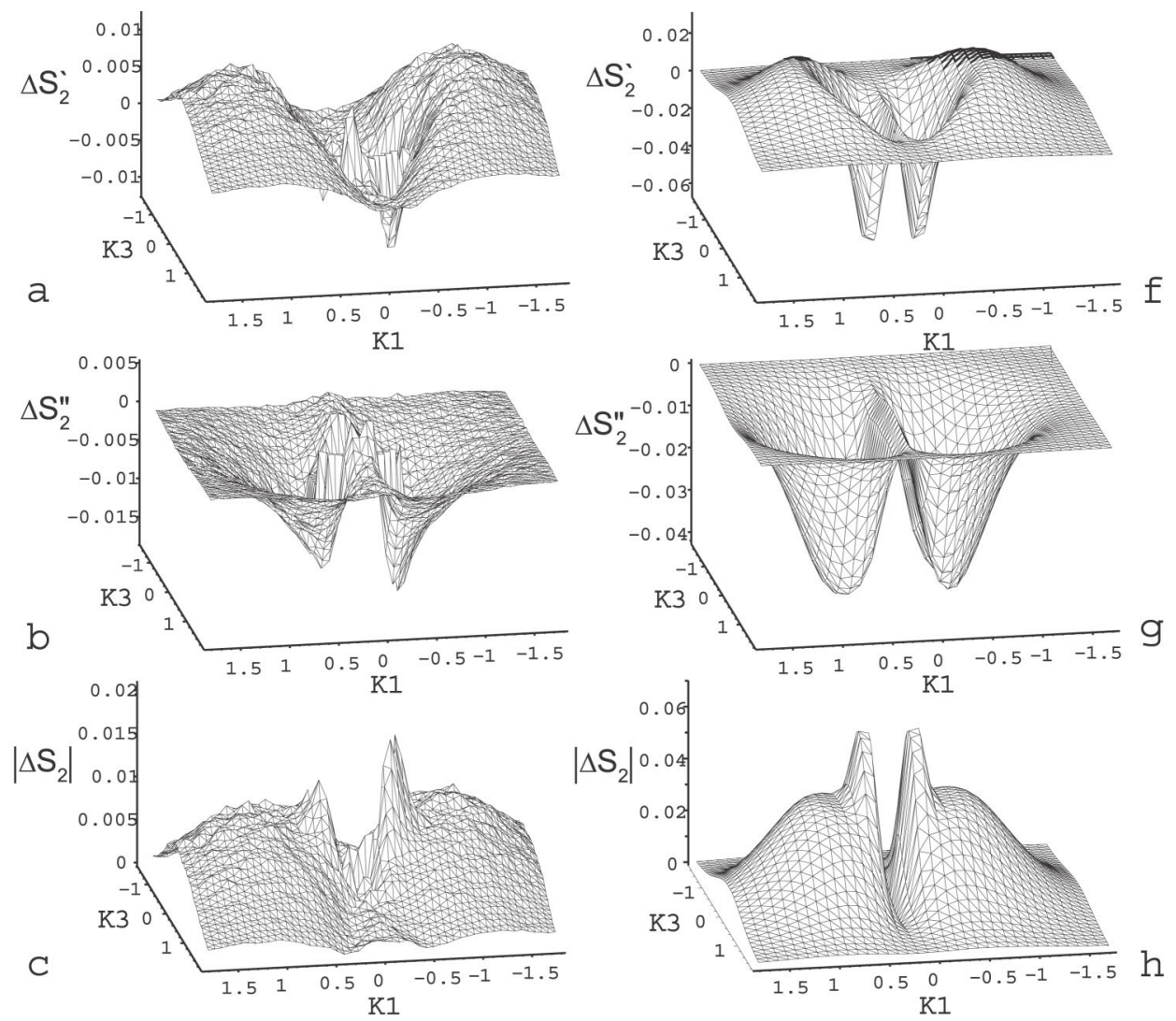

FIG. 10. The various Fourier components of the shear-distorted structure factor for experiment (a) to (e) and theory (f) to (j). The experimental data were taken for $\dot{\gamma}_{0}=0.68 \mathrm{~s}^{-1}$ and $\omega=2 \pi 0.05 \mathrm{~s}^{-1}$, with $\xi=945 \mathrm{~nm}$. The corresponding theoretical values are calculated using $\lambda / \dot{\gamma}=0.6$.
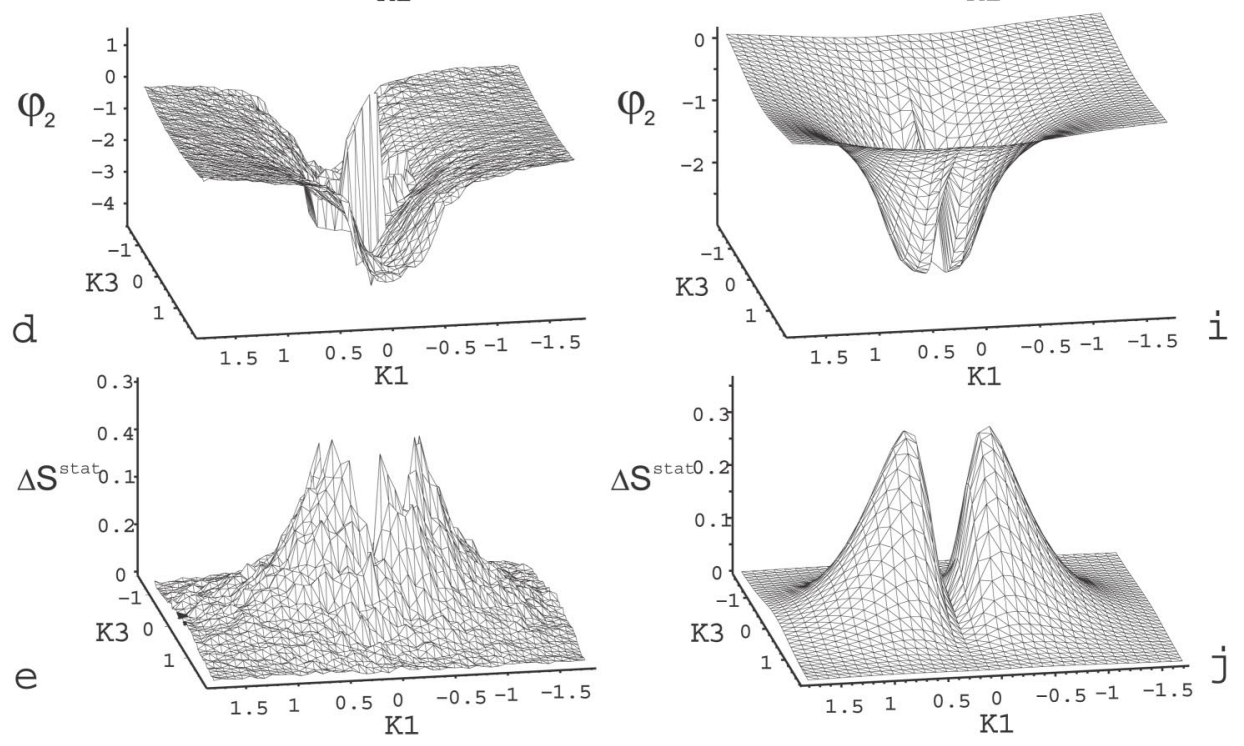

sured by the appearance of higher order harmonics in the frequency domain of the response, as depicted in Fig. 9(b). Comparing the amplitudes of the harmonics for the different shear rates, we see that the fourth order harmonic appears when doubling the maximum shear rate from $\dot{\gamma}_{0}$ $=0.29 \mathrm{~s}^{-1}$ to $\dot{\gamma}_{0}=0.77 \mathrm{~s}^{-1}$. When the maximum shear rate is doubled once again to $\dot{\gamma}_{0}=1.43 \mathrm{~s}^{-1}$, also the sixth order harmonic of the response becomes significant. The fit to the theory reproduces this increasing nonlinearity with increasing shear rate. From the Fourier analysis also the phase shift of the microstructural response with respect to the applied field is obtained. The phase shift decreases from $\varphi$
$=1.78 \mathrm{rad}$ for $\dot{\gamma}_{0}=0.29 \mathrm{~s}^{-1}$, to $\varphi=0.47 \mathrm{rad}$ for $\dot{\gamma}_{0}=0.77 \mathrm{~s}^{-1}$, and $\varphi=-0.33$ rad for $\dot{\gamma}_{0}=1.43 \mathrm{~s}^{-1}$.

Varying the frequency at a fixed shear rate one expects two phenomena to occur. First, one expects the imaginary component to grow and the real component to diminish with increasing frequency. Second, one expects the degree of nonlinearity to decrease with increasing frequency.

Both phenomena can be observed in Fig. 12, where cuts are shown of the real and imaginary components of the structure factor at $K_{3}=0$. The peak values of the real component decrease and move outward while the peak values of the imaginary components increase and move inward with in- 


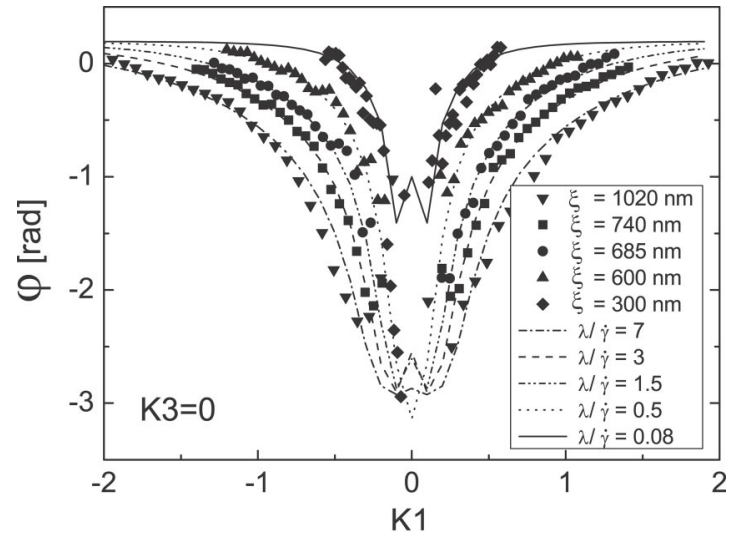

FIG. 11. The $K_{1}$ dependence of the phase shift for several correlation lengths for $\omega=2 \pi 0.5 \mathrm{~s}^{-1}$ and $\dot{\gamma}_{\max }=1.0 \mathrm{~s}^{-1}$. The symbols are experimental data, and the lines are fits with respect to the proportionality constant $\lambda / \dot{\gamma}$, which is the only fit parameter. The results of the fit are plotted in Fig. 6, and are seen to coincide with values obtained from stationary shear experiments.

creasing frequency, for both the second and fourth harmonic. When plotting the wave-vector-dependent phase shift (see Fig. 13), an increasing time lag is observed with increasing frequency. This implies that for increasing frequency an increasing amount of energy is stored at small wave vectors of the critical structure, instead of distorting the critical structure. The theoretical predictions are qualitatively confirmed by the experiments, though the fourth harmonic data are quite noisy.

In order to compare the experimental values for $\Delta S$ with theory, the experimental data are scaled with a constant to fit the theoretical prediction. Interestingly, this constant, and therefore the mismatch with theory, decreases with increasing frequency, from 4.5 to 2 , so that the quality of the fit improves with increasing frequency. Apart from the scaling, also the positions of the maxima in $\Delta S(\mathbf{K})$ do not quantitatively agree with the theoretical predictions. It is striking to see that for the second harmonic the maxima of the second harmonic for the experiment occur at larger $K_{1}$ values as predicted by theory, whereas for the fourth harmonic they occur at smaller $K_{1}$ values, even within the experimental beam stop. Also the maxima of the imaginary components are found at very small $K$ values, within the region covered by the beam stop. The phase shifts, however, are correctly predicted by theory (see Fig. 13), showing that the timedependent behavior is recovered by the theory.

\section{SUMMARY AND DISCUSSION}

The aim of this work is to gain microscopic understanding of the behavior of near-critical suspensions under shear flow. We tested the theory for the evolution of the probability density function under shear, by measuring in situ the sheardistorted microstructure of colloid-polymer dispersions.

Under stationary shear flow, an unexpected shear-induced distortion of near-critical microstructural order in directions perpendicular to the flow direction was observed. A quantitative explanation for the experimentally observed distortion

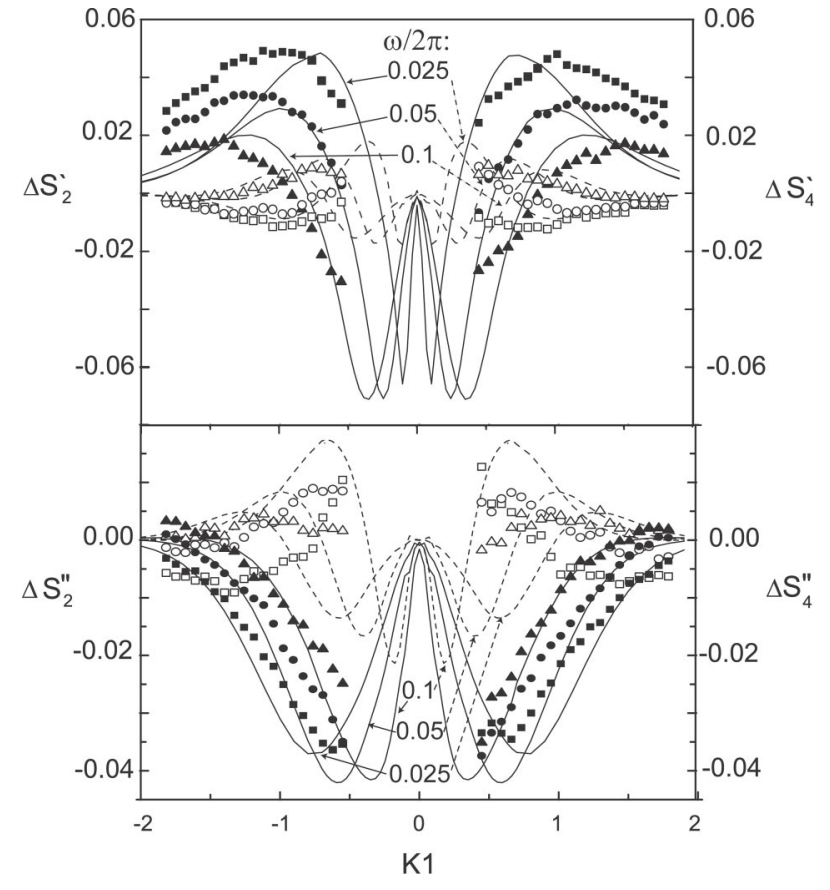

FIG. 12. Frequency dependence of the shear-distorted structure factor for $\xi=945 \mathrm{~nm}$ and a fixed shear rate $\dot{\gamma}_{0}=0.8 \pm 0.1 \mathrm{~s}^{-1}$, with $K_{3}=0$. Plotted are the amplitudes of the second and fourth harmonics of the real (top) and imaginary (bottom) parts of the response. The solid symbols represent the second component and the open symbols represent the fourth component of the experimental response, for $\omega=2 \pi 0.025 \mathrm{~s}^{-1}(\boldsymbol{\Delta}), \omega=2 \pi 0.05 \mathrm{~s}^{-1}(\boldsymbol{\bullet})$, and $\omega$ $=2 \pi 0.1 \mathrm{~s}^{-1}(\boldsymbol{\square})$. The solid and dashed lines indicate the theoretical predictions of the second and fourth components, respectively, using $\lambda / \dot{\gamma}=0.6$ to calculate $\Omega$. The experimental data are multiplied by a factor 4.5 for $\omega=2 \pi 0.025 \mathrm{~s}^{-1}, 3.0$ for $\omega=2 \pi 0.05 \mathrm{~s}^{-1}$, and 2.0 for $\omega=2 \pi 0.1 \mathrm{~s}^{-1}$.

of long-ranged correlations is only feasible when the distortion of short-ranged correlations is properly taken into account in theory. Scattering patterns in the flow-vorticity plane and turbidity for a whole set of equilibrium correlation lengths (that is, the distance to the critical point before shearing) and shear rates could be quantitatively described in terms of just three independent fitting parameters: one parameter describing the susceptibility of the long-ranged correlations and two parameters describing the susceptibility

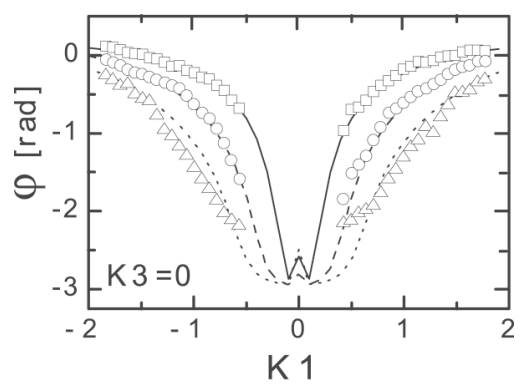

FIG. 13. Frequency dependence of the phase shift for a fixed shear rate $\left(\dot{\gamma}_{0}=0.8 \pm 0.1 \mathrm{~s}^{-1}\right)$ with $K_{3}=0: \omega=2 \pi 0.025 \mathrm{~s}^{-1}(\square), \omega$ $=2 \pi 0.05 \mathrm{~s}^{-1}(\bigcirc), \omega=2 \pi 0.1 \mathrm{~s}^{-1}(\triangle)$. The lines indicate the theoretical prediction, using $\lambda / \dot{\gamma}=0.6$ to calculate $\Omega$. 
of the short-ranged correlations. The shear-rate and equilibrium-correlation-length dependence of these parameters is found to be in accordance with theory.

On first thought one might reason that short-ranged distortions are unimportant when probing the much more susceptible long-ranged, near-critical correlations. It is shown, however, that close to the critical point such distortions of short-ranged correlations do play an important role. The structure factor is of the Ornstein-Zernike form in directions perpendicular to the flow direction, which allows one to define a shear-rate-dependent correlation length. By probing the structure factor in the flow direction we observe that the correlation length is always smaller than the correlation length without shear flow. This is a proof of a shear-induced displacement of the critical point. The fact that the data for stationary shear can be fitted by assuming a distortion of the short-ranged correlations confirms the theoretical prediction that a shear-induced displacement of the critical point is connected to distortions of short-ranged correlations [42].

Short-ranged correlations are less important when explaining experimental scattering data under oscillatory flow, probably due to the fact that short-ranged microstructural order instantaneously follows the imposed shear flow. For a (semi)quantitative comparison of the data to theory we had to introduce, however, an unexplained additional phase shift to the temporal response of the near-critical structure factor, and we had to normalize scattered intensities. The theory then explains experimental results on a (semi)quantitative level: all the characteristic features of oscillatory shearinduced microstructural order as a function of shear rate, maximum strain amplitude, frequency, and equilibrium correlation length, are explained by our theory using the dressed Peclet number as a single fit parameter.

The description of shear-induced short-ranged correlations has been done in a semiempirical manner. In particular, the nonanalytic shear-rate dependence that we need to explain the experiments is not yet understood. Probably there is a coupling of the nonlinear response of long-ranged correlations and the response of short-ranged distortions. A combination of light scattering (to probe long-ranged distortions) and x-ray scattering (to probe short-ranged distortions [8]) on the same system may give insight into the origin of the nonanalytic shear-rate dependence of short-ranged correlations close to the critical point. To confirm that our theory indeed predicts viscoelastic behavior of near-critical dispersions correctly, we need to perform Fourier-transform rheology, which is suitable to study nonlinear behavior of such dispersions [50].

\section{ACKNOWLEDGMENT}

We acknowledge Tjerk Lenstra for the synthesis of the colloidal particles and the preparation of the critical sample.
[1] A. Onuki, J. Phys.: Condens. Matter 9, 6119 (1997).

[2] P. Butler, Curr. Opin. Colloid Interface Sci. 4, 214 (1999).

[3] J. Vermant, Curr. Opin. Colloid Interface Sci. 6, 489 (2001).

[4] V. A. Tolpekin, M. H. G. Duits, D. van den Ende, and J. Mellema, Langmuir 20, 2614 (2004).

[5] H. Hoekstra, J. Vermant, and J. Mewis, Langmuir 19, 9134 (2003)

[6] P. Varadan and M. J. Solomon, Langmuir 17, 2918 (2001).

[7] L. B. Chen, M. K. Chow, B. J. Ackerson, and C. F. Zukoski, Langmuir 10, 2817 (1994).

[8] P. Panine, T. Narayanan, J. Vermant, and J. Mewis, Phys. Rev. E 66, 022401 (2002).

[9] M. F. Butler and M. Heppenstall-Butler, Food Hydrocolloids 17, 815 (2003).

[10] C. K. Chan, F. Perrot, and D. Beysens, Phys. Rev. A 43, 1826 (1991).

[11] T. Hashimoto, K. Matsuzaka, Elisha Moses, and A. Onuki, Phys. Rev. Lett. 74, 126 (1995).

[12] J. Lauger, Ch. Laubner, and Wolfram Gronski, Phys. Rev. Lett. 75, 3576 (1995).

[13] A. H. Krall, J. V. Sengers, and K. Hamano, Phys. Rev. Lett. 69, 1963 (1992).

[14] L. Berthier, Phys. Rev. E 63, 051503 (2001).

[15] A. J. Bray, A. Cavagna, and R. D. M. Travasso, Phys. Rev. E 65, 016104 (2002).

[16] F. Corberi, G. Gonnella, and A. Lamura, Phys. Rev. Lett. 81, 3852 (1998).

[17] A. Onuki, Phys. Rev. E 55, 403 (1997).
[18] J. K. G. Dhont and G. Nägele, Phys. Rev. E 58, 7710 (1998).

[19] J. K. G. Dhont and N. J. Wagner, Phys. Rev. E 63, 021406 (2001).

[20] D. Beysens, M. Gbadamassi, and L. Boyer, Phys. Rev. Lett. 43, 1253 (1979).

[21] D. Beysens, M. Gbadamassi, and B. Moncef-Bouanz, Phys. Rev. A 28, 2491 (1983).

[22] A. I. Nakatani, H. Kim, Y. Takahashi, Y. Matsushita, A. Takano, B. J. Bauer, and C. C. Han, J. Chem. Phys. 93, 795 (1990).

[23] E. K. Hobbie, D. W. Hair, A. I. Nakatani, and C. C. Han, Phys. Rev. Lett. 69, 1951 (1992).

[24] E. K. Hobbie, A. I. Nakatani, H. Yajima, J. F. Douglas, and C. C. Han, Phys. Rev. E 53, R4322 (1996).

[25] X.-L. Wu, D. J. Pine, and P. K. Dixon, Phys. Rev. Lett. 66, 2408 (1991).

[26] P. K. Dixon, D. J. Pine, and X.-L. Wu, Phys. Rev. Lett. 68, 2239 (1992).

[27] J. W. van Egmond, D. E. Werner, and G. G. Fuller, J. Chem. Phys. 96, 7742 (1990).

[28] S. Fujii, T. Isojima, and K. Hamano, Phys. Lett. A 263, 393 (1999).

[29] H. Verduin and J. K. G. Dhont, Phys. Rev. E 52, 1811 (1995).

[30] I. Bodnár, H. Verduin, and J. K. G. Dhont, Phys. Rev. Lett. 77, 5304 (1996).

[31] I. Bodnár and J. K. G. Dhont, Phys. Rev. Lett. 80, 1662 (1998).

[32] J. K. G. Dhont and H. Verduin, J. Chem. Phys. 101, 6193 
(1994).

[33] T. A. J. Lenstra, Z. Dogic, and J. K. G. Dhont, J. Chem. Phys. 114, 10151 (2001).

[34] T. Sun, A. C. Balazs, and D. Jasnow, Phys. Rev. E 59, 603 (1999).

[35] S. Butler and P. Harrowell, Nature (London) 415, 1008 (2002).

[36] G. K. Batchelor, J. Fluid Mech. 83, 97 (1977).

[37] J. F. Brady, J. Chem. Phys. 99, 567 (1993).

[38] R. A. Lionberger and W. B. Russel, J. Chem. Phys. 106, 402 (1997).

[39] G. Szamel, J. Chem. Phys. 114, 8708 (2001).

[40] N. J. Wagner and W. B. Russel, Physica A 155, 475 (1988).

[41] H. Wang, M. P. Lettinga, and J. K. G. Dhont, J. Phys.: Condens. Matter 14, 7599 (2002).
[42] J. K. G. Dhont, Phys. Rev. Lett. 76, 4269 (1996).

[43] S. Asakura and F. Oosawa, J. Chem. Phys. 22, 1255 (1954).

[44] A. Vrij, Pure Appl. Chem. 48, 471 (1976).

[45] J. K. G. Dhont, An Introduction to Dynamics of Colloids (Elsevier, Amsterdam 1996).

[46] J. M. Deutch and I. J. Oppenheim, J. Chem. Phys. 54, 3547 (1971).

[47] T. J. Murphy and J. L. Aguirre, J. Chem. Phys. 57, 2098 (1972).

[48] M. Fixman, Adv. Chem. Phys. 6, 175 (1964).

[49] T. A. J. Lenstra and J. K. G. Dhont, Phys. Rev. E 63, 061401 (2000).

[50] M. Wilhelm, D. Maring, and H. W. Spiess, Rheol. Acta 37, 399 (1998). 